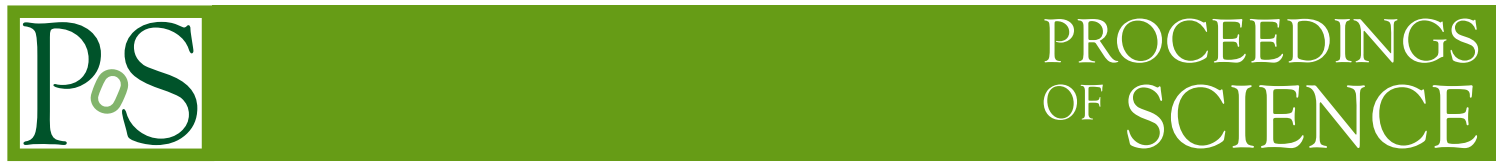

\title{
Grand unification and CP violation
}

\author{
Jihn E. Kim*淿 \\ Center for Axion and Precision Physics Research (CAPP, IBS), 291 Daehakro, Yuseong-Gu, \\ Daejeon 34141, Republic of Korea, and \\ Department of Physics, Kyung Hee University, 26 Gyungheedaero, Dongdaemun-Gu, Seoul \\ 02447, Republic of Korea \\ E-mail: jihnekimegmail.com
}

I will talk on my recent works related to the flavor grand unification, weak $\mathrm{CP}$ violation, and the phases in the CKM and PMNS matrices.

38th International Conference on High Energy Physics

3-10 August 2016

Chicago, USA

\footnotetext{
*Speaker.

${ }^{\dagger}$ This work is supported in part by the National Research Foundation (NRF) grant funded by the Korean Government (MEST) (NRF-2015R1D1A1A01058449) and the IBS (IBS-R017-D1-2016-a00).
} 


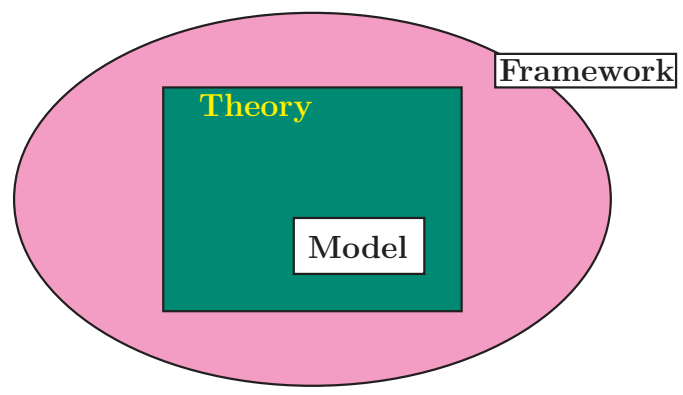

Figure 1: Gross's thought about the framework-theory-model relation.

\section{Introduction}

In this talk, I will concentrate on the mere $5 \%$ of the energy pie (mainly of atoms) of the Universe. In grand unification theories(GUTs), it is the property of chiral fermions, forbidding large masses due to the chiral symmetry. For the flavor solution in GUTs, the GUT symmetry has to be extended. Nowadays, flavor symmetries are studied mainly by some discrete symmetries, due to the observed large mixing angles in the leptonic sector. But, flavor symmetry may be a gauge symmetry in which case a true unification is GUTs with the flavor symmetry included there. The first attempt along this line was due to Georgi in SU(11) [1] on the unification of GUT families (UGUTF). The next try with spinor representation of $\mathrm{SO}(4 n+2)$ groups was in $\mathrm{SO}(14)$ [2]. My attempt along this line in the last year was from string compactification [3] based on $\mathbf{Z}_{12-I}$ orbifold compactification.

Recently, Gross presented a cartoon on our attempts that we are interested in [4]. There is a grand "Framework" which is quite general, acceptable to all scientists. That may include quantum mechanics or symmetry. It may include very interesting "Theory" such as Einstein's gravity and string theory, as shown in Fig. 1. Within this theory, one can build a "Model" which must be a working example. Even though the design, "Framework", is fantastic, without a model example some will say that it is a religion. So, our efforts is to find a working model toward the theory/framework design. In this vein, we attempt to understand CP violation and GUTs in particle physics and cosmology.

To discuss violation of a symmetry, first one has to define the symmetry. Even though kinetic mixings of $\mathrm{U}(1)$ gauge bosons have been considered for some time, the definition is usually done such that the kinetic energy terms are diagonal. In the standard model(SM), the kinetic energy terms of quarks, leptons, and Higgs doublets are $\mathrm{CP}$ conserving. The $\mathrm{CP}$ violation in the SM arises in the interaction terms, typically through the Yukawa couplings. If the VEVs of Higgs doublets vanish, then there is no CP violation because all fermions are massless. Below the VEV scale of the Higgs doublets, all the SM fields obtain masses, and one can locate the CP phase in the left-handed currents, coupling to $W_{\mu}^{ \pm}$. The charged current couplings are defined in this setup and the CKM and PMNS matrices are defined respectively for quarks and leptons. The CKM and PMNS matrices are unitary, which is the only condition for the CKM and PMNS matrices, and so there are many different parametrization schemes [5]. Because the CKM matrix elements are rather well-known by now, there are three classes of parametrizations [6], each having the same CP phase $\delta_{\mathrm{CKM}}$. 
The CP violation in the SM is an interference phenomenon, encompassing all three families. This will become clearer below when we express the Jarlskog determinant $J$.

\section{Jarlskog phases in the CKM and PMNS matrices}

The discussion on the strong $\mathrm{CP}$ is not separable from the discussion of the weak CP violation. Nowadays, the strong CP problem is well understood in "invisible" axions [7]. So, I will concentrate on the weak $\mathrm{CP}$ here.

Recently, it has been pointed out that a new parametrization of the CKM matrix $V_{\mathrm{CKM}}$ (三 $V$ below) with one row (or column) real is very useful to scrutinize the physical effects of the weak $\mathrm{CP}$ violation. Then, the elements of the determinant directly give the weak $\mathrm{CP}$ phase [5]. The physical significance of the weak CP violation is given by the Jarlskog determinant $J$ [8] which is obtained from the imaginary part of a product of two elements of $V$ and two elements of $V^{*}$ of the CKM matrix, e.g. of the type $V_{12} V_{23} V_{13}^{*} V_{22}^{*}$. This Jarlskog determinant is just twice the area of the Jarlskog triangle. In Ref. [9], we have shown that one easily obtains the Jarlskog determinant from the entries of $V$ if Det. $V=1$. If Det. $V \neq 1$, one can make it so by multiplying an overall phase to all the up- or all the down-type quarks. We obtained $J=\left|\operatorname{Im} V_{31} V_{22} V_{13}\right|$. By looking at the above triple product form on $J$, we can see where the physical CP phase appears, and in terms of the Kim-Seo(KS) parametrization angles [5],

$$
J=\left|c_{1} c_{2} c_{3} s_{1}^{2} s_{2} s_{3} \sin \delta_{\mathrm{CKM}}\right|,
$$

where $s_{1}=\sin \theta_{1}$, etc. The above form of $J$ includes all mixing angles, i.e. all three families must parcitipate in the diagram for weak $\mathrm{CP}$ violation. $\mathrm{CP}$ violation is an interference phenomenon. Note that the unitarity triangle in the PDG book gives [10]

$$
\text { PDG15: } \alpha=\left(85.4_{-3.8}^{+3.9}\right)^{\mathrm{o}}, \beta=\left(21.50_{-0.74}^{+0.76}\right)^{\mathrm{o}} \gamma=\left(68.0_{-8.5}^{+8.0}\right)^{\mathrm{o}} \text {. }
$$

The recent UTfit gives [11],

$$
\text { UTfit16: } \alpha=(88.6 \pm 3.3)^{\circ}, \beta=(22.03 \pm 0.86)^{\circ}, \gamma=(69.2 \pm 3.4)^{\circ} \text {, }
$$

and the recent CKMfit gives with the unitarity constraint over the world average [12]

$$
\text { CKMfit16: } \alpha=\left(90.6_{-1.1}^{+3.9}\right)^{\mathrm{o}}, \beta=\left(24.21_{-1.35}^{-1.33}\right)^{\mathrm{o}}, \gamma=\left(66.9_{-3.44}^{+0.94}\right)^{\mathrm{o}} \text {. }
$$

So, there are three possibilities for the $\mathrm{CP}$ phase from our formula on $J: \delta_{\mathrm{CKM}}=\alpha, \beta$, or $\gamma$. Note that $\alpha$ is close to $\frac{\pi}{2}$ in Eqs. $(2.2,2.3,2.4)$. Make Det=1 as in the KS form [5]. Then, we observe that

1. Make the real part of (22) element very large as in many parametrizations. Then,

$$
\delta_{\mathrm{CKM}}=\alpha
$$

The parametrizations in Ref. [5] give $\delta_{\mathrm{CKM}}=\alpha$. Also, the Kobayashi-Maskawa form gives (2.5), by multiplying a universal phase since its determinant is not real. 
2. If both 1 st row $=$ real and 1 st column $=$ real, then the Chau-Keung parametrization gives

$$
\delta_{\mathrm{CKM}}=\gamma
$$

Also, the Maiani parametrization gives (2.6), by multiplying a universal phase.

So, it is proved that $J=\left|\operatorname{Im} . V_{31} V_{22} V_{13}\right|$ is very useful.

We can use the KS parametrization to show the maximality of the weak CP violation. For this, we use the fact that any Jarlskog triangle has the same area. With the $\lambda=\sin \theta_{C}$ expansion, the area of the Jarlskog triangle is of order $\lambda^{6}$. For the triangle with two long sides of order $\lambda$. by rotating the $O\left(\lambda^{5}\right)$ side, one can show that the area is maximum for $\delta \simeq \frac{\pi}{2}$, and the maximality $\delta=\frac{\pi}{2}$ is a physical statement. The same must be true even if we use the CKM parametrization, but the proof on the maximality around the observed real parameters may not be so simple.

Within this scheme, we cannot determine which parametrization is more useful over the others. There must be another independent condition to choose a proper parametrization.

In the leptonic sector also, there is a preliminary hint that $\delta_{\mathrm{PMNS}} \neq 0$, and close to $-\frac{\pi}{2}$ even though the error bar is large [13]. The quark mixing angles are $\theta_{i}$ and $\delta_{\mathrm{CKM}}$, and lepton mixing angles are $\Theta_{i}$ and $\delta_{\mathrm{PMNS}}$. Even if $\theta_{i}$ and $\Theta_{i}$ cannot be related, we can relate $\delta_{\mathrm{CKM}}$ and $\delta_{\mathrm{PMNS}}$ if there is only one CP phase in the whole theory. Indeed, this has been shown in Ref. [14] where the weak $\mathrm{CP}$ violation is spontaneous and one unremovable phase is located at the weak interaction singlet $a$ la the Froggatt-Nielsen(FN) mechanism [15]. In the supersymmetric model, it was shown that one phase in the ultra-violet completion gives [6]: $\delta_{\mathrm{PMNS}}= \pm \delta_{\mathrm{CKM}}$. Then, the Jarlskog triangles of the quark and lepton sectors will have one common phase.

There are many phases in concern: CKM, PMNS, Majorana, and leptogenesis phases. If there is only one phase, all of these must be expressed in terms of one phase. So, the Majorana phase determined at the intermediate scale and the leptogenesis phase can be also expressed in terms of this one phase, as shown in Ref. [16].

One obvious strategy to relate the CKM and PMNS phases is GUTs, but the CP phase is a property of families. This leads us to the consideration of UGUTF as commented in Introduction. There are the constraint from the FCNC, i.e. the family symmetry breaking scale is above $10^{5} \mathrm{GeV}$.

In a talk in the flavor physics parallel session here, theories on mixing angles were classified as [17]

(i) $\mathrm{SM} \times($ family symmetry),

(ii) GUT $\times$ (family symmetry),

(iii) Unification of GUT families in a simple gauge group,

where the family symmetry was considered as a discrete group such as $A_{4}$. In Fig. 2, the direct product of gauge and flavor symmetries are shown. In Table 1, the flavor group $G_{f}$ is further classified depending on discrete and continuous possibilities. These classifications belong to Items (i) and (ii). In the remainder of my talk, however, I concentrate on Item (iii), where $G_{g} \times G_{f}$ is in a simple group.

As commented in Introduction, we discuss UGUTFs. In string compactification, there is one example $\mathrm{SU}(7) \times \mathrm{U}(1)$ from $\mathbf{Z}_{12-I}$ orbifold compactification [3], and the possibility for one $\mathrm{CP}$ phase is also obtained in [6]. 


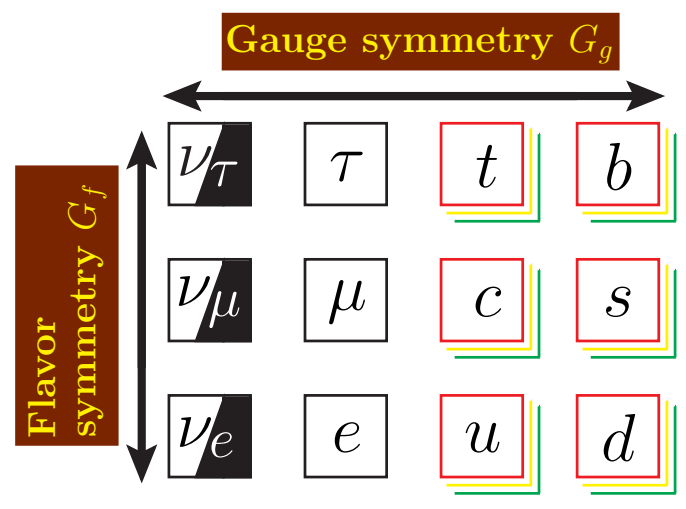

Figure 2: A cartoon for $G_{g} \times G_{f}$ with three colors of quarks.

\begin{tabular}{|c|c|c|}
\hline & Continuous & Discrete \\
\hline Abelian & $\mathrm{U}(1)$ & $\mathbf{Z}_{N}$ \\
\hline Non-Abelian & $\mathrm{SU}(3), \mathrm{SO}(3), \cdots$ & $A_{4}, S_{4}, A_{5}, \Delta(48), \cdots$ \\
\hline
\end{tabular}

Table 1: A classification of $G_{f}[17]$.

In the unification of families in $\mathrm{SU}(N)$ GUTs, $\mathrm{SU}(5)$ families are counted by the number of $10 \mathrm{~s}$ [1]. For example, a few antisymmetric representations of $\mathrm{SU}(N)$ allow the following family numbers: $\mathrm{SU}(5): \Psi^{[\alpha \beta]}, n_{f}=1 ; \mathrm{SU}(6): \Psi^{[\alpha \beta \gamma]}, n_{f}=0, \Psi^{[\alpha \beta]}, n_{f}=1 ; \mathrm{SU}(7): \Psi^{[\alpha \beta \gamma]}, n_{f}=$ 1, $\Psi^{[\alpha \beta]}, n_{f}=1$. With this kind of counting, Ref. [1] in SU(11) with $\Psi^{[\alpha \beta \gamma \delta]} \oplus \Psi_{[\alpha \beta \gamma]} \oplus \Psi_{[\alpha \beta]} \oplus$ $\Psi_{[\alpha]}$ obtained three $\mathrm{SU}(5)$ families. In string compactification, it is very difficult to obtain large $\mathrm{SU}(N)$ groups. For example, the rank 8 group $\mathrm{E}_{8}$ cannot allow a subgroup with rank greater than 8. Reference [3] obtained a UGUTFs of three families in $\mathrm{SU}(7) \times \mathrm{U}(1)$, a kind of flipped form. The SU(7) representations are a $\Psi^{[\alpha \beta \gamma]}$ from the untwisted sector and two $\Psi^{[\alpha \beta]}$ s from the twisted sector. Thus, it gives three families.

In the model of [3], it has been shown that there is a possibility of $\delta_{\mathrm{PMNS}}= \pm \delta_{\mathrm{CKM}}$ [18] if only one CP phase is present in the GUT scale VEVs. Since the discussion is more involved there, we refer to a simpler study [14].

\section{Conclusion}

My talk on weak CP is centered on flavor unification, emphasizing the possibility in GUTs. A few emphases were: (1) The Jarlskog determinant $J$ is $\left|\operatorname{Im} V_{31} V_{22} V_{13}\right|$ in the KS form, (2) it is shown that the Jarlskog determinant $J$ is almost maximum with the current determination of quark (real) mixing angles. There is a possibility that $\delta_{\mathrm{CKM}}$ can be $\frac{\pi}{2}$, which is the case in the KS [5] and Kobayashi-Maskawa forms of the CKM matrix, (3) There is a possibility that $\delta_{\text {PMNS }}$ can be maximal [13], (4) Unification of GUT families is possible with $\mathrm{SU}(7) \times \mathrm{U}(1)$, which is derived in $\mathbf{Z}_{12-I}$ orbifold compactification [3], and and (5) with the FN singlets it is possible to relate the CKM, PMNS, Majorana, and leptogenesis phases. 


\section{References}

[1] H. Georgi, Towards a grand unified theory of flavor, Nucl. Phys. B 156 (1979) 126 [doi: 10.1016/0550-3213(79)90497-8].

[2] J.E. Kim, Model of flavor unity, Phys. Rev. Lett. 45 (1980) 1916 [doi: 10.1103/PhysRevLett.45.1916].

[3] J. E. Kim, Toward unity of families: anti-SU(7) from $\mathbf{Z}_{(12-I)}$ orbifold compactification, JHEP 1506 (2015) 114 [arXiv: 1503.03104].

[4] D.J. Gross, talk presented at PASCOS 2016, Quy N'hon, June 10-16, 2016.

[5] J. E. Kim and M-S. Seo, Parametrization of the CKM matrix, Phys. Rev. D 84 (2011) 037303 [arXiv:1105.3304[hep-ph]], and references therein.

[6] J. E. Kim and S. Nam, Unifying CP violations of quark and lepton sectors, Euro. Phys. J. C 75 (2015) 619 [arXiv:1506.08491v4 [hep-ph]], and [arXiv:1506.08491v2].

[7] For my recent reviews, see, J.E. Kim, CP-conservation in QCD and why only "nvisible” axions work, Talk presented at 12th PATRAS Conference, Jeju Island, Korea, 20-24 June 2016 [arXiv: 1608.07256 [hep-ph]].

[8] C. Jarlskog, Commutator of the quark mass matrices in the standard electroweak model and a measure of maximal CP violation, Phys. Rev. Lett. 55 (1985) 1039 [doi: 10.1103/PhysRevLett.55.1039].

[9] J. E. Kim, D. Y. Mo, and S. Nam, Final state interaction phases using data from CP asymmetries, J. Korean Phys. Soc. 66 (2015) 894 [arXiv:1402.2978].

[10] A. Ceccucci, Z. Ligeti, and Y. Sakai, The CKM quark mixing matrix, in K.A. Olive et al.(Particle Data Group), Chin. Phys. C38 (2014) 090001.

[11] A. Pich, Flavor physics, Lecture presented at Corfu Summer School and Workshop on Standard Model and Beyond, Corfu, Greece, Sep. 2, 2016.

[12] N. Harnew, Measuring the unitarity triangle with LHCb, Talk presented at Corfu Summer School and Workshop on Standard Model and Beyond, Corfu, Greece, Sep. 3, 2016.

[13] S.V. Cao, Recent neutrino oscillation results from T2K, Talk presented at PASCOS 2016, Quy N'hon, June 10-16, 2016.

[14] J. E. Kim, The CKM matrix with maximal CP violation from $\mathbf{Z}_{12}$ symmetry, Phys. Lett. B 704 (2011) 360 [arXiv:1109.0995[hep-ph]].

[15] C.D. Froggatt and H.B. Nielsen, Hierarchy of quark masses, Cabibbo angles and CP violation, Nucl. Phys. B 147 (1979) 277 [doi: 10.1016/0550-3213(79)90316-X].

[16] L. Covi, J. E. Kim, B. Kyae, and S. Nam, Leptogenesis with high-scale electroweak symmetry breaking and an extended Higgs sector, Phys. Rev. D 94 (2016) 065004 [doi: 10.1103/PhysRevD.94.065004].

[17] Y-L. Zhou, Theoretical motivations for the precision measurement of the oscillation parameters, Talk presented here on Aug 4, 2016.

[18] J. E. Kim, D. Y. Mo, and M-S. Seo, The CKM matrix from anti-SU(7) unification of GUT families, Phys. Lett. B 749 (2015)) 476 [arXiv:1506.08984 [hep-ph]]. 